\title{
Active energy management strategies for active distribution system
}

\author{
Yue XIANG ${ }^{1}$, Junyong LIU $^{1}$, Wei YANG ${ }^{1}$, Cheng HUANG ${ }^{2}$
}

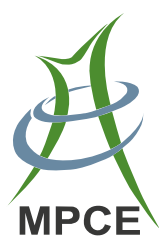

\begin{abstract}
Active energy management is an effective way to realize the flexible utilization of distributed energy resources to suit the characteristics of active distribution system. Advanced active energy management strategies need to be designed to coordinate the optimization of 'generation, network, load'. An active management model is built for the local distribution system integrated with the generation curtailment mechanism and the charging/discharging management of plug-in electric vehicles. Furthermore, different strategies based on the energy management model are presented. The model and strategies are tested and discussed in a modified distribution system, and the impacts with different load profiles are also analyzed.
\end{abstract}

Keywords Active distribution system, Renewable generation, Plug-in electric vehicles, Active management

CrossCheck date: 22 September 2015

Received: 9 April 2015/Accepted: 22 September 2015/Published online: 31 October 2015

(C) The Author(s) 2015. This article is published with open access at Springerlink.com

$\triangle$ Yue XIANG

eexxyy@126.com

Junyong LIU

liujy@scu.edu.cn

Wei YANG

yangwei_scu@126.com

Cheng HUANG

opcodesec@gmail.com

1 School of Electrical Engineering and Information, Sichuan University, Chengdu 610065, China

2 College of Electronics and Information Engineering, Sichuan University, Chengdu 610065, China

\section{Introduction}

Active distribution system (ADS) is proposed to be a solution for realizing the flexible operation and maximizing the utilization of assets [1-3], which is driven by the low carbon target and the diversity of the distributed resources (DERs), such as distributed generation (DG), distributed storage and flexible load.

Besides the opportunities, the essential challenges, for example, the energy management of ADS, have attracted the interest of researchers in recent years. In terms of DG, although it is clean and low-carbon, the randomness and intermittency of renewable generation always bring new challenges in the operation phase. Infrastructure upgrades could be used to help expand the capacity of DG integration, and effective management with existing assets is another economic option. For example, an effective reconfiguration method was deployed in [4] to accommodate larger amounts of DG in distribution systems without network reinforcement. An advanced distribution network management system was also presented in [5] aiming at maximizing wind energy harvesting while simultaneously managing congestion and voltages. However, a basic assumption, i.e., the power generation of DGs following the generic maximum power point tracking (MPPT) mode, is always made in most of the previous studies of economic operation of the distribution system. It is reasonable to take full use of DGs without violating the distribution network constraints, so as to promote the utilization of the renewable energy in the early stage. However, with the increase of the penetration level, appropriate generation curtailment would become inevitable to guarantee the security operation of the power system. Specifically, when the generation production of a feeder surpasses its consumption, a reverse power flow occurs which might lead to over-voltage and causes problems to the coordination of 
protective devices [6]. Thus, renewable energy should be treated as a manageable resource, that the power output can be restricted or curtailed, in the future flexible operation of the active distribution system instead of operating in an absolute MPPT mode, to achieve comprehensive benefit from the system perspective.

Additionally, distributed storage system (DSS) provides a feasible way to cooperate the 'uncertain' renewable generation and load, improving the asset utility efficiency. In [79], DSS was effectively integrated with the distribution network to realize the economic operation of the system. In recent years, transportation electrification is a hot topic for the various benefits associated with electric vehicles, especially the plug-in electric vehicle (PEV), which directly absorbs energy from the power system for propulsion. The proliferation of PEVs brings power industry opportunities and challenges. On one hand, the uncontrolled charging load may occur coincidentally with the peak hours because of the consuming habit, which will affect or even deteriorate the operation performance of power systems dramatically [1012]. On the other hand, since most of the PEVs are in the idle for most of time [13], so charging load is possible to be managed as a kind of flexible resources. Additionally, with the development of vehicle to grid (V2G) technology, power can be bidirectional-flow between the distribution system and PEVs. Thus, PEVs can be regarded as a kind of distributed mobile storage resources, and participate in the active energy management.

It can be seen from the existing studies that either the generation curtailment control or PEV management is mainly used as a reserve resource to alleviate the pressure of the distribution system and guarantee security. This paper mainly focuses on the active management of the local distribution system, and investigates the active management strategy for the ADS from the view of achieving the maximum economic benefit for the local power company, not only guarantee the security itself but also take the full advantage of the renewable energy and flexible charging/discharging behavior of PEVs.

In Sect. 2, the active management model is proposed firstly. Then the PEVs' behavior is modeled and different strategies are summarized and presented. In Sect. 3, simulations and discussions are given on the test system. And the conclusions are drawn in Sect. 4.

\section{Problem formulation}

\subsection{Basic active management model}

In terms of the local distribution system, the energy management is designed and realized by the interaction among distribution system operator (DSO), the aggregators and the dispatching center of the upper grid. An aggregator represents a cluster of the same DERs connected to the same bus, which can be an equivalent model for the specific DERs. The basic simple active management procedures are designed as follows: (1) Aggregators integrate DERs' information, such as the forecasted renewable power, and send it to DSO; (2) After receiving the information from aggregators and the upper grid, DSO makes the coordinated optimal schedule and sends back to them. It is noted that, for simplicity, aggregators and DSO are considered belonging to the local power company in the same area. So it is assumed that there is no transaction fee between them. In this way, cooperated with the aggregator agents, DSO is considered to be able to modify the operation of devices that are connected to its network. Therefore, considering the renewable integration and power purchased from the upper grid, the basic active management model is formulated to maximize the economic benefits for the local power company as

$\max \left(U_{\mathrm{L}}-C_{\mathrm{DG}}-C_{\mathrm{G}}\right)$

where $U_{\mathrm{L}}$ is the economic benefits from power supply for the residential loads; $C_{\mathrm{DG}}$ is the generation cost for the aggregated DGs; $C_{\mathrm{G}}$ is the cost for purchasing power from the upper grid.

$U_{\mathrm{L}}=\sum_{i=1}^{N} \sum_{t=1}^{T} B_{t}^{\mathrm{L}} P_{i, t}^{\mathrm{L}} x_{i}^{\mathrm{L}}$

$C_{\mathrm{DG}}=\sum_{i=1}^{N} \sum_{t=1}^{T} B^{\mathrm{DG}}\left(P_{i, t}^{\mathrm{DG}}-\Delta P_{i, t}^{\mathrm{DG}}\right) x_{i}^{\mathrm{DG}}$

$C_{\mathrm{G}}=\sum_{t=1}^{T} B_{t}^{\mathrm{G}} P_{t}^{\mathrm{G}}$

where $N$ is the total number of the buses; $T$ is the total dispatching period; $B_{t}^{\mathrm{L}}$ is the electricity price in time period $t ; P_{i, t}^{\mathrm{L}}$ is the resident active load demand at bus $i$ in time period $t ; x_{i}^{\mathrm{L}}$ is the binary state for indicating whether there exists residential load demand at bus $i$, if so, $x_{i}^{\mathrm{L}}=1 ; B^{\mathrm{DG}}$ is the unit operation price of $\mathrm{DG} ; P_{i, t}^{\mathrm{DG}}$ is the forecasted power of DG at bus $i$ in time period $t ; \Delta P_{i, t}^{\mathrm{DG}}$ is the curtailed power of DG at bus $i$ in time period $t ; x_{i}^{\mathrm{DG}}$ is the binary state for indicating whether there exists $\mathrm{DG}$ at bus $i$, if so, $x_{i}^{\mathrm{DG}}=1$; $B_{t}^{\mathrm{G}}$ is the unit purchasing price from the upper grid; $P_{t}^{\mathrm{G}}$ is the power purchased from the upper grid in time period $t$.

The power balance constraints are

$$
\begin{aligned}
P_{i, t}^{\mathrm{G}} x_{i}^{\mathrm{G}}+ & \left(P_{i, t}^{\mathrm{DG}}-\Delta P_{i, t}^{\mathrm{DG}}\right) x_{i}^{\mathrm{DG}}-P_{i, t}^{\mathrm{L}} x_{i}^{\mathrm{L}} \\
& =V_{i, t} \sum_{j=1}^{N} V_{j, t}\left(g_{i j} \cos \theta_{i j, t}+b_{i j} \sin \theta_{i j, t}\right)
\end{aligned}
$$


$Q_{i, t}^{\mathrm{G}} x_{i}^{\mathrm{G}}-Q_{i, t}^{\mathrm{L}} x_{i}^{\mathrm{L}}=V_{i, t} \sum_{j=1}^{N} V_{j, t}\left(g_{i j} \sin \theta_{i j, t}-b_{i j} \cos \theta_{i j, t}\right)$

where $P_{i, t}^{\mathrm{G}}$ is the power purchased from the upper grid at bus $i$ in time period $t ; x_{i}^{\mathrm{G}}$ is the binary state for indicating whether bus $i$ connects the active distribution system to the upper grid, if so, $x_{i}^{\mathrm{G}}=1 ; Q_{i, t}^{\mathrm{L}}$ is the resident reactive load demand at bus $i$ in time period $t ; V_{i, t}$ and $V_{j, t}$ are the voltage magnitude at bus $i$ and $j$ in time period $t$, respectively; $Q_{i, t}^{\mathrm{G}}$ is the reactive power from the upper grid at bus $i$ in time period $t ; g_{i j}$ is the conductance of branch $i-j ; b_{i j}$ is the susceptance of branch $i-j ; \theta_{i j, t}$ is the difference of phase angle between bus $i$ and bus $j$ in time period $t$.

The voltage magnitude limit and power flow limit of each branch are

$\underline{V} \leq V_{i, t} \leq \bar{V}$

$\left|P_{i j, t}\right| \leq P_{i j}^{\max }$

where $\underline{V}$ is the lower bound of allowable voltage magnitude; $\bar{V}$ is the upper bound of allowable voltage magnitude; $P_{i j}^{\max }$ is the power flow limit of branch $i-j ; P_{i j, t}$ is the power flow of branch $i-j$ in time period $t$.

The power limit from the upper grid is formulated as

$0 \leq P_{i, t}^{\mathrm{G}} \leq \bar{P}^{\mathrm{G}}$

where $\bar{P}^{\mathrm{G}}$ is the maximum power purchased from the upper grid in one time period.

\subsection{PEV modeling}

The modeling of PEV behavior is the basis for the energy management with PEV, as well as charging and discharging control. For the sake of transportation, the charging/discharging behavior is based on owner habits and trip needs, so the behavior is featured with stochastic valuation and the time for active energy management is limited. However, in a certain resident district [11, 14], large-scale stochastic trips of PEVs can be statistically described with the probability distribution according to their daily habits. Thus, it is possible to simulate the trip information of large-scale PEVs.

Firstly, some assumptions are given: (1) All the PEVs controlled by the aggregators have the same kind of batteries and can be operated in a smart charging and discharging mode with contracts; (2) Each PEV owner is independent from other PEV owners, and the charging/ discharging time aligns with the owner' habit. When the PEV is plugged in, the owner has to specify the departure time and total charging energy required (or expected); (3) Private cars are mainly involved since this paper focuses on smart management of electric vehicles in the residential district (the parking lots).

According to [15-17], the last time of the trip (arriving time), the first time of the trip (departure time), and the initial battery state (the battery state of charge (SOC) when it arrivals at the parking slot, which can be estimated according to its mileage) can be generated by the probability density functions (PDFs), and formulated as (10)-(12), respectively.

$f^{\mathrm{ARR}}(x)=\left\{\begin{array}{c}\frac{1}{\sigma_{\mathrm{ARR}} \sqrt{2 \pi}} \exp \left[-\frac{\left(x+24-\mu_{\mathrm{ARR}}\right)^{2}}{2 \sigma_{\mathrm{ARR}}^{2}}\right] \\ 0<x \leq \mu_{\mathrm{ARR}}-12 \\ \frac{1}{\sigma_{\mathrm{ARR}} \sqrt{2 \pi}} \exp \left[\begin{array}{c}\left.-\frac{\left(x-\mu_{\mathrm{ARR}}\right)^{2}}{2 \sigma_{\mathrm{ARR}}^{2}}\right] \\ \mu_{\mathrm{ARR}}-12<x \leq 24\end{array}\right.\end{array}\right.$

where $\mu_{\mathrm{ARR}}$ and $\sigma_{\mathrm{ARR}}$ are the mean value and standard deviation of arrival time PDF and $\mu_{\mathrm{ARR}}=17.5$, $\sigma_{\mathrm{ARR}}=3.4$.

Equation (10) is used to describe the distribution that the PEV arrives at the parking slot, i.e., the last time for the trip in Day 1. Then, the possible arrival time $t_{\text {arr0 }}^{m}$ of the $m^{\text {th }}$ PEV can be simulated based on the distribution.

$f^{\mathrm{DEP}}(x)=\left\{\begin{array}{r}\frac{1}{\sigma_{\mathrm{DEP}} \sqrt{2 \pi}} \exp -\left[\frac{\left(x-\mu_{\mathrm{DEP}}\right)^{2}}{2 \sigma_{\mathrm{DEP}}^{2}}\right] \\ 0<x \leq \mu_{\mathrm{DEP}}+12 \\ \frac{1}{\sigma_{\mathrm{DEP}} \sqrt{2 \pi}} \exp \left[-\frac{\left(x-\mu_{\mathrm{DEP}}-24\right)^{2}}{2 \sigma_{\mathrm{DEP}}^{2}}\right] \\ \mu_{\mathrm{DEP}}+12<x \leq 24\end{array}\right.$

where $\mu_{\mathrm{DEP}}$ and $\sigma_{\mathrm{DEP}}$ are the mean value and standard deviation of departure time PDF and $\mu_{\mathrm{DEP}}=8.9$, $\sigma_{\mathrm{DEP}}=3.2$.

Equation (11) can describe the distribution that the PEV departures from the parking slot, i.e., the first time for the trip in Day 2. Then, the possible departure time of the $m^{\text {th }}$ PEV can be simulated, as $t_{\text {dep } 0}^{m}$.

$f_{m}(x)=\frac{1}{\sigma_{m} x \sqrt{2 \pi}} \exp \left[-\frac{\left(\ln x-\mu_{m}\right)^{2}}{2 \sigma_{m}^{2}}\right]$

where $\mu_{m}=2.98 ; \sigma_{m}=1.14$; the energy efficiency of each $\mathrm{PEV}$ is $0.15 \mathrm{kWh} / \mathrm{km}$.

Since the dispatching time period is set from 12:00 in Day 1 to 12:00 in Day 2 in this paper, time interval label transformation would be implemented according to (13) and (14), respectively.

$$
\begin{aligned}
& t_{\text {arr }}^{m}=\left\{\begin{array}{cc}
1 & t_{\text {arr } 0}^{m} \in[0: 00,12: 00] \\
t_{\text {arr } 0}^{m}-12+1 & \text { else }
\end{array}\right. \\
& t_{\text {dep }}^{m}=\left\{\begin{array}{cc}
25 & t_{\text {dep } 0}^{m} \in[12: 00,24: 00] \\
t_{\mathrm{dep} 0}^{m}+12+1 & \text { else }
\end{array}\right.
\end{aligned}
$$


where $t_{\mathrm{arr}}^{m}$ and $t_{\mathrm{dep}}^{m}$ are the corresponding arrival and departure time in the programming, respectively. For simplicity, it is assumed that the arrival time is limited to reflect the time from 12:00 to 24:00 in Day 1, and the departure time is from 0:00 to 12:00 in Day 2. For example, if the $m^{\text {th }}$ PEV arrives at the parking slot at 18:00 in Day 1, $t_{\text {arr }}^{m}=7$.

\subsection{Active management strategies}

Besides the renewable DGs in Sect. 2.1, PEVs are used as one of the resources in the energy management framework of ADS. Thus, the energy management framework can be extended as follows: aggregators integrate PEVs' information, e.g., SOC, charging or discharging power, and transfer an equivalent model upstream to DSO. Then, DSO makes the coordinated optimal schedule to be combined with all the information received from all kinds of aggregators and the upper grid. It is noted that, when the PEV in the parking slots plugs to the grid, it would communicate with the corresponding aggregator and follow the schedule received until departure. Thus, the objective function of the active management will be updated as

$\max \left(U_{\mathrm{L}}-C_{\mathrm{DG}}-C_{\mathrm{G}}+J^{\mathrm{EV}}\right)$

where $J^{\mathrm{EV}}$ is the economic benefits from PEVs.

Different strategies can be implemented based on the proposed model, to investigate the dispatching schedules of DERs based on different energy management goals. Regarding the DGs, the forecasted value is obtained according to the natural conditions, e.g., wind speed for the WT and solar intensity for the PV. However, in real operation, the outputs are reduced, for the sake of the network constraints and dispatching schedules. In that way, compared with the MPPT mode, it is similar to 'lose' a part of the forecasted power, which is described as 'curtailment' in this paper. The generation curtailment mechanism will effectively work in the active management model. For the participating of PEVs, when $\bar{P}_{m}^{\mathrm{CH}}\left(t_{\mathrm{dep}}^{m}-t_{\mathrm{arr}}^{m}\right) \geq E_{m, t_{\mathrm{dep}}^{m}}^{\mathrm{EXP}}$ $-E_{m, t_{\text {arr }}^{m}}$, the charging or discharging process can be managed. $\bar{P}_{m}^{\mathrm{CH}}$ is the upper limit of charging power; $E_{m, t_{\text {arr }}^{m}}$ is the SOC of the $m^{\text {th }}$ PEV's battery when it arrives; $E_{m, t_{\text {dep }}^{m}}^{\mathrm{EXP}}$ is the expected SOC when it departures. If not, it will not participate in the flexible control and be charged with $\bar{P}_{m}^{\mathrm{CH}}$ during the time period $\left[t_{\mathrm{arr}}, t_{\mathrm{dep}}\right)$. According to the energy management framework described, three strategies are summarized and designed to be tested.

1) S1: Non-interruptible charging of PEVs

The discharging mode is not considered in this strategy, so the economic benefit from the PEVs is detailed as
$J^{\mathrm{EV}}=\sum_{m=1}^{M} \sum_{t=1}^{T} B^{\mathrm{CH}} P_{m, t}^{\mathrm{CH}}$

where $B^{\mathrm{CH}}$ is the unit charging price; $P_{m, t}^{\mathrm{CH}}$ is the charging power of the $m^{\text {th }} \mathrm{PEV}$ in time period $t ; M$ is the total equivalent PEV number.

In this strategy, the charging process of all of the PEVs starts with $\bar{P}_{m}^{\mathrm{CH}}$ at the moment when arriving till disconnected from the distribution network or the SOCs satisfy the expectations, and no interruptions would occur when the network constraints complied. Additionally, related constraints should also be respected:

$E_{m, t+1}=E_{m, t}+P_{m, t}^{\mathrm{CH}} \Delta t$

$E_{m, t} \leq \bar{E}_{m}$

$P_{m, t}^{\mathrm{CH}}=\left\{\begin{array}{cl}\bar{P}_{m}^{\mathrm{CH}} & t \in\left[t_{\mathrm{arr}}, t_{\mathrm{dep}}\right) \& E_{m, t-1}<\sigma \bar{E}_{m} \\ 0 & \text { else }\end{array}\right.$

$$
\begin{gathered}
P_{i, t}^{\mathrm{G}} x_{i}^{\mathrm{G}}+\left(P_{i, t}^{\mathrm{DG}}-\Delta P_{i, t}^{\mathrm{DG}}\right) x_{i}^{\mathrm{DG}}-P_{i, t}^{\mathrm{L}} x_{i}^{\mathrm{L}}-\sum_{m=1}^{M_{i}} P_{m, i, t}^{\mathrm{CH}} x_{i}^{\mathrm{E}} \\
=V_{i, t} \sum_{j=1}^{N} V_{j, t}\left(g_{i j} \cos \theta_{i j, t}+b_{i j} \sin \theta_{i j, t}\right)
\end{gathered}
$$

where $E_{m, t}$ is the SOC of the $m^{\text {th }}$ PEV's battery in time period $t ; \bar{E}_{m}$ is the upper boundary of SOC for the sake of the battery life span; $\sigma$ is the expected ratio when departure; $\Delta \mathrm{t}$ is the time interval, $M_{i}$ is the total equivalent $\mathrm{PEV}$ number at bus $i ; x_{i}^{\mathrm{E}}$ is the binary state for indicating whether there exists PEV aggregator at bus $i$, if so, $x_{i}^{\mathrm{E}}=1$.

Therefore, the models in S1 include (2-9), (15)-(20).

2) S2: Intelligent charging of PEVs

Compared with $\mathrm{S} 1$, the PEVs charging process of this strategy is much more flexible, which can be optimally scheduled according to (15), as long as it does not violate the network constraints and satisfies the expectation.

$0 \leq P_{m, t}^{\mathrm{CH}} \leq \bar{P}_{m}^{\mathrm{CH}} \quad$ if $\quad \bar{P}_{m}^{\mathrm{CH}}\left(t_{\mathrm{dep}}^{m}-t_{\mathrm{arr}}^{m}\right) \geq E_{m, t_{\mathrm{dep}}^{m}}^{\mathrm{EXP}}-E_{m, t_{\mathrm{arr}}^{m}}$

$P_{m, t}^{\mathrm{CH}}=\bar{P}_{m}^{\mathrm{CH}} \quad$ if $\quad \bar{P}_{m}^{\mathrm{CH}}\left(t_{\mathrm{dep}}^{m}-t_{\mathrm{arr}}^{m}\right) \leq E_{m, t_{\mathrm{dep}}^{m}}^{\mathrm{EXP}}-E_{m, t_{\mathrm{arr}}^{m}}$

$P_{m, t}^{\mathrm{CH}}=0 \quad t \notin\left[t_{\mathrm{arr}}, t_{\mathrm{dep}}\right)$

$P_{m, t}^{\mathrm{CH}} \Delta t \leq \eta^{\mathrm{CH}}\left(\bar{E}_{m}-E_{m, t}\right)$

$E_{m, t_{\mathrm{dep}}}=E_{m, t_{\mathrm{dep}}^{m}}^{\mathrm{EXP}}=\sigma \bar{E}_{m} \quad$ if $\quad \bar{P}_{m}^{\mathrm{CH}}\left(t_{\mathrm{dep}}^{m}-t_{\mathrm{arr}}^{m}\right) \geq E_{m, t_{\mathrm{dep}}^{m}}^{\mathrm{EXP}}-E_{m, t_{\mathrm{arr}}^{m}}$

Equation (21) indicates that PEVs can be charged in the time period $\left[t_{\text {arr }}, t_{\text {dep }}\right.$ ) with flexible charging power between zero and maximal charging power to satisfy PEVs' expectations. If the $\mathrm{PEV}$ does not participate in the flexible control, i.e., it cannot reach the expected SOC 
when departure, it would be charged with $\bar{P}_{m}^{\mathrm{CH}}$ during the time period $\left[t_{\mathrm{arr}}, t_{\mathrm{dep}}\right.$ ), as illustrated in (22). $\eta^{\mathrm{CH}}$ is charging limitation ratio. In this way, it provides DSO the ability to arrange PEVs charging with DG resources and power purchased from the upper gird to maximize the benefits based on the active management model with (2)-(4), (6)(9), (15)-(18) and (20)-(25).

3) S3: Intelligent charging and discharging of PEVs

In this strategy, discharging mode is included in the active management framework, so the economic benefit from the PEVs is detailed as

$J^{\mathrm{EV}}=\sum_{m=1}^{M} \sum_{t=1}^{T}\left(B^{\mathrm{CH}} P_{m, t}^{\mathrm{CH}}-B^{\mathrm{DCH}} P_{m, t}^{\mathrm{DCH}}\right)$

where $B^{\mathrm{DCH}}$ is the unit discharging compensation cost, i.e., the unit battery loss cost caused by discharging, which is formulated in (27) [17-19]; $P_{m, t}^{\mathrm{DCH}}$ is the discharging power of the $m^{\text {th }} \mathrm{PEV}$ in time period $t$.

$B^{\mathrm{DCH}}=\frac{C_{\mathrm{B}}}{L_{\mathrm{C}} E_{\mathrm{B}} D}$

where $C_{\mathrm{B}} / E_{\mathrm{B}}$ is the unit purchasing cost of the battery; $L_{\mathrm{C}}$ is recycle amount in discharging; $D$ is discharging deep when $L_{\mathrm{C}}$ is tested.

The charging/discharging state of PEV can only be one of the three states, e.g., charging, discharging or dull, in a time interval, which should satisfy (28).

$P_{m, t}^{\mathrm{CH}} P_{m, t}^{\mathrm{DCH}}=0$

$0 \leq P_{m, t}^{\mathrm{DCH}} \leq \bar{P}_{m}^{\mathrm{DCH}} \quad$ if $\quad \bar{P}_{m}^{\mathrm{CH}}\left(t_{\mathrm{dep}}^{m}-t_{\mathrm{arr}}^{m}\right) \geq E_{m, t_{\mathrm{dep}}^{m}}^{\mathrm{EXP}}-E_{m, t_{\mathrm{arr}}^{m}}$

$P_{m, t}^{\mathrm{DCH}}=0 \quad$ if $\quad \bar{P}_{m}^{\mathrm{CH}}\left(t_{\mathrm{dep}}^{m}-t_{\mathrm{arr}}^{m}\right) \leq E_{m, t_{\mathrm{dep}}^{m}}^{\mathrm{EXP}}-E_{m, t_{\text {arr }}^{m}}$

$P_{m, t}^{\mathrm{DCH}}=0 \quad t \notin\left[t_{\mathrm{arr}}, t_{\mathrm{dep}}\right)$

$P_{m, t}^{\mathrm{DCH}} \Delta t \leq \eta^{\mathrm{DCH}}\left(E_{m, t}-\underline{E}_{m}\right)$

where $\bar{P}_{m}^{\mathrm{DCH}}$ is the upper limit of discharging power; $\eta^{\mathrm{DCH}}$ is the discharging limitation ratio.

Besides, the other constraints need to be updated:

$$
\begin{aligned}
& E_{m, t+1}=E_{m, t}+P_{m, t}^{\mathrm{CH}} \Delta t-P_{m, t}^{\mathrm{DCH}} \Delta t \\
& \underline{E}_{m} \leq E_{m, t} \leq \bar{E}_{m} \\
& P_{i, t}^{\mathrm{G}} x_{i}^{\mathrm{G}}+\left(P_{i, t}^{\mathrm{DG}}-\Delta P_{i, t}^{\mathrm{DG}}\right) x_{i}^{\mathrm{DG}}-P_{i, t}^{\mathrm{L}} x_{i}^{\mathrm{L}} \\
& \quad-\sum_{m=1}^{M_{i}}\left(P_{m, i, t}^{\mathrm{CH}}-P_{m, i, t}^{\mathrm{DCH}}\right) x_{i}^{\mathrm{E}} \\
& \quad=V_{i, t} \sum_{j=1}^{N} V_{j, t}\left(g_{i j} \cos \theta_{i j, t}+b_{i j} \sin \theta_{i j, t}\right)
\end{aligned}
$$

where $\underline{E}_{m}$ is the lower boundary of SOC.

The models in S3 include (2)-(4), (6)-(9), (15), (21)(23) and (26)-35). Due to the discharging mode integrated in the active management model, PEVs can act as distributed storage systems which can output power back to the ADS to compensate the conventional peak load when the electricity price is very high and renewable generation capacity is limited. The essence of PEV integration in the energy management is to realize the coordinated optimization with its interruptible charging/discharging mechanism to improve the 'active' of the ADS based on the proposed active management model and strategies.

In addition, different load profiles would affect the scheduling of the energy management with the active management strategies, which are also studied and tested with the generic ELEXON load profile classes [20] in the following cases.

\section{Case study}

\subsection{Case description}

In this paper, the modified UKGDS-EHV network 1 [21] is adopted as the test system. It contains 61 buses with weekly loops and has three base voltage levels $(132,33$ and $11 \mathrm{kV}$ ), as shown in Fig. 1. The part in dashed line is the

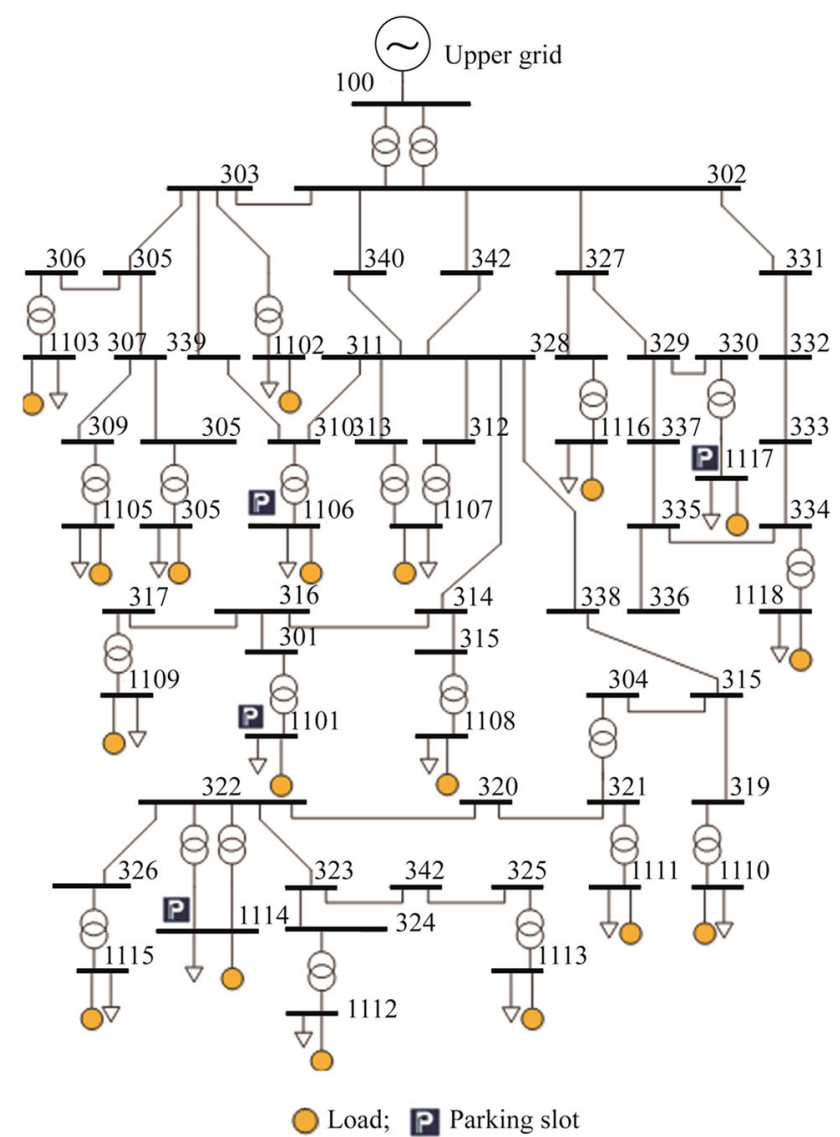

Fig. 1 Test system 
local energy system, operated by the local power company through DSO and aggregators. Bus 100 is set as the balance bus with the active power range $[0,60] \mathrm{MW}$, connected with the upper grid. The load profile in the maximum operation mode is listed in [21]. It is designed that each load bus is equipped with a PV aggregation generation, and the aggregated installation capacity approximately equals to the load demand at the same bus. The dispatching periods are from 12:00 in Day 1 to 12:00 in Day 2. The nominal coefficient templates for PV and resident load demand in each time period are shown in Fig. 2. The network parameters, such as the power flow limit of each branch can be found in [21]. Let $B^{\mathrm{PV}}=0.18 \mathrm{Yuan} / \mathrm{kWh}$. Time-of-use electricity price [22] is adopted in the system, and the detailed information could be found in Table 1. Let $B_{t}^{\mathrm{G}}=0.8 B_{t}^{\mathrm{L}}, \eta^{\mathrm{DCH}}=0.9, \eta^{\mathrm{CH}}=0.9$.

Four equivalent parking lots (aggregators) are introduced at the $11 \mathrm{kV}$ level buses, as shown in Fig. 1. The numbers of PEVs can be controlled by the aggregators are 3000, 1500, 1000 and 2000 at Buses 1106, 1101, 1117 and 1114, respectively. In each parking lot, it is assumed that the owner of the PEV has signed the contract that allows the batteries of his own vehicle to be dispatched if it is plugged in. The charging/discharging power limit is set as $3 \mathrm{~kW}$, while the total capacity of each PEV is $20 \mathrm{kWh}$. Let the expected SOC when departure be $19 \mathrm{kWh}$. Let $C_{\mathrm{B}} /$ $E_{\mathrm{B}}=500($ Yuan $/ \mathrm{kWh}), D=0.8, L_{\mathrm{c}}=1000$, thus $B^{\mathrm{DCH}}$ can be obtained as 0.625 Yuan/kWh based on (27), and let $B^{\mathrm{CH}}=0.6$ Yuan $/ \mathrm{kWh}$. For the additional aggregated power injection from PEVs, the loading rate of the original system needs to be updated. Considering the extreme

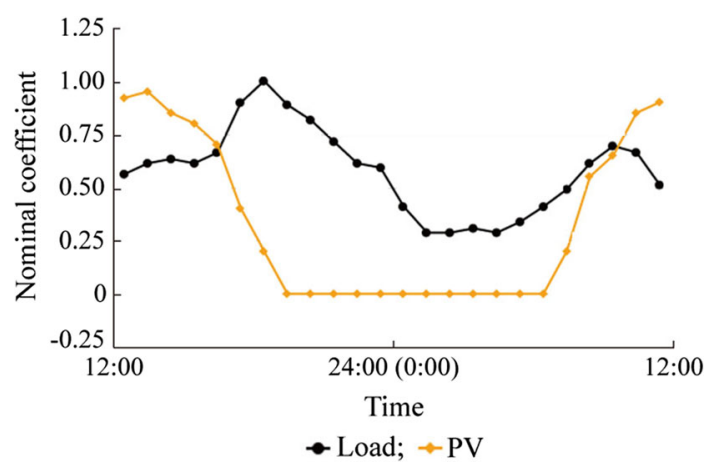

Fig. 2 Nominal coefficient template

Table 1 Time-of-use electricity price

\begin{tabular}{ll}
\hline Time & Price (Yuan/kWh) \\
\hline 23:00-7:00 & 0.27 \\
7:00-8:00, 12:00-17:00, 22:00-23:00 & 0.49 \\
8:00-12:00, 17:00-22:00 & 0.83 \\
\hline
\end{tabular}

condition of all PEVs' integration, the power flow limit at Branches 330-1117, 322-1114, 301-1101 and 310-1106 are updated as 5, 9, 7 and $11 \mathrm{MW}$, respectively.

For simplicity, every 10 PEVs are aggregated together, i.e., initial SOC conditions and the energy management schedule will be the same. So, 750 groups of PEVs' related variables are generated in the programming. According to (10)-(14), Monte Carlo simulation is adopted. In order to avoid the overcharge and over-discharge, SOC of batteries is limited within $[1,19] \mathrm{kWh}$. The generated arrival time, departure time and the initial SOC conditions are shown in Figs. 3, 4, respectively.

Programs are designed and implemented in the GAMS platform [23] using a Celeron E3300 $2.5 \mathrm{GHz} / 1.96 \mathrm{~GB}$ computer.

\subsection{Simulation of active management strategy}

Three scenarios are simulated based on the proposed active management model and the corresponding strategies in Sect. 2. The energy management results are output to the designed web-based visualization platform, as shown in Figs. 5, 6 and 7, respectively. The total economic benefits in the three scenarios are listed in Table 2.

The PVs would be the priority resource to be scheduled to balance the loads because of the lower operation cost, however, the curtailment may occur in the following two situations. Firstly, during valley load period when the PV generation surpasses the load, the surplus part has to be curtailed due to there is no power to the upper gird assumed in this paper. Secondly, the network operation constraints limit the full utilization of the PVs, which make it inevitable to curtail the power generated by the PVs to maintain the distribution system operate safely. If the outputs of PVs cannot satisfy all load demands, the remaining would be compensated by other power generation resources, such as power from the upper gird. As seen from the figures, the forecasted PV power curves depicted

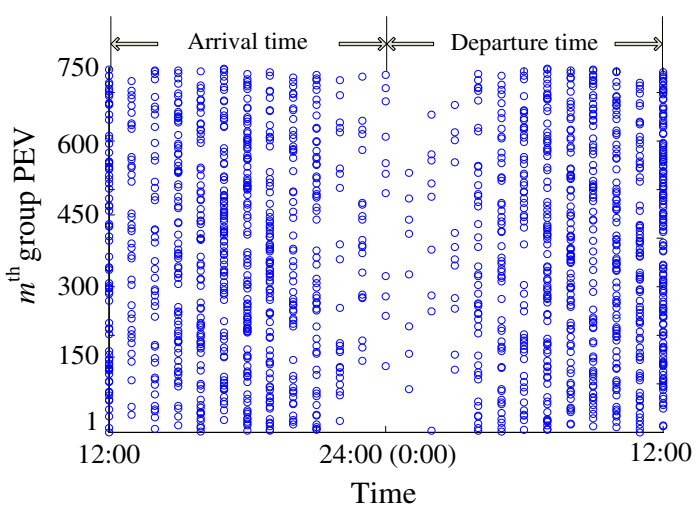

Fig. 3 Generated arrival time and departure time of PEVs 


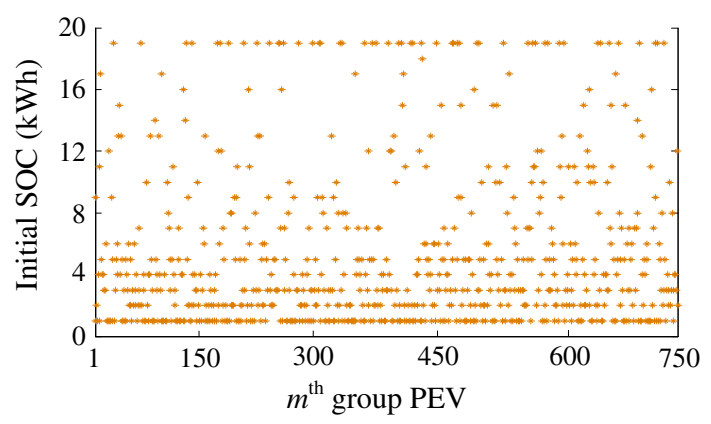

Fig. 4 Generated initial SOC of PEVs

by the orange dash lines reflect the forecasted value according to the natural conditions of solar within the dispatching time horizon. While the orange solid lines illustrate the real generation power, and parts of the power of PV have been curtailed.

Figure 5 illustrates the outcomes of active management with $\mathrm{S} 1$ that the charging process of PEV is non-interruptible, and it starts to charge whenever arriving if needed. There are 694 PEVs to be charged during the dispatching horizon in this case. As shown in Fig. 5, the charging load grows with more and more PEVs to be pluged in when people arrive home from work, and it is not until time period 16:00-17:00, when the load demand is larger than the forecasted PV generation, there is no curtaiment in PV generation. The power purchased from the upper grid increases from time period 15:00-16:00 and eventually becomes the dominate power source, with the PV generation decreases to zero gradually due to getting dark. And from the time period 7:00-8:00 in Day 2, the power purchased from the upper grid decreases for the power generated by PV increases. In the intelligent charging mode, when $\mathrm{S} 2$ is adopted, the charging load of PEVs is distributed among the dispatching horizons, as shown in Fig. 6. And the power purchased from the upper grid is altered as well, which has a smaller peak compared with S1. The economic benefit obtained is 166695 Yuan, and $4 \%$ more than that in S1, as shown in Table 2. The scenario is illustrated in Fig. 7 when the discharging mode is considered. There are 747 PEVs involved in the active management process, and an obvious pattern, that the equivalent charging process moves to the midnight and the next early morning when the residential load and electricity price are low, can be observed in Fig. 7. The price purchasing power the upper grid from time 17:00 to 24:00 is higher than discharging cost, so PEVs are scheduled to discharge to compensate the peak load without violating the constraints of the PEVs. And the distribution company can get the most benefit, $14.89 \%$ more compared with that in S1, when $\mathrm{S} 3$ is adopted.

The SOC evolution of each PEV when S2 and S3 are adopted is shown in Figs. 8 and 9, respectively, where the darker the color is, the smaller the SOC would be. As we can see, the black parts on both sides represent that the PEVs have not arrived or have left already. It is obvious that there exists more white area in S2 than S3 from 21:00

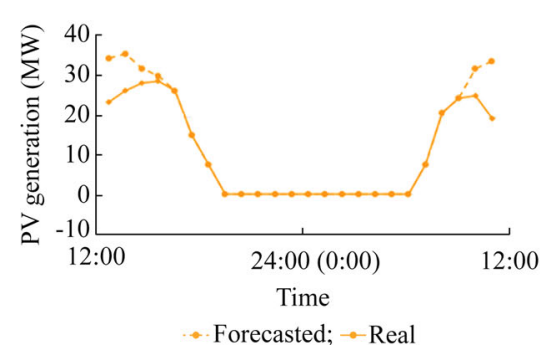

Fig. 5 Energy management results with S1
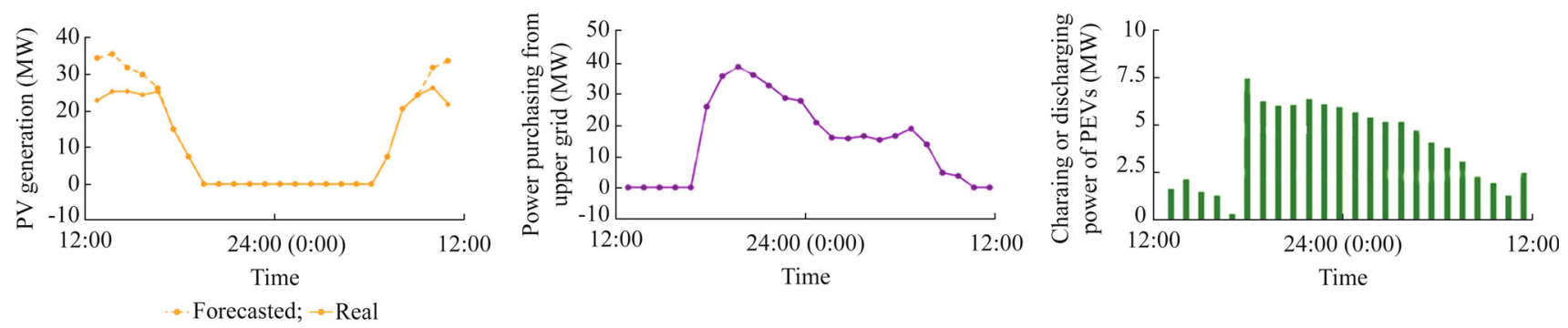

Fig. 6 Energy management results with S2
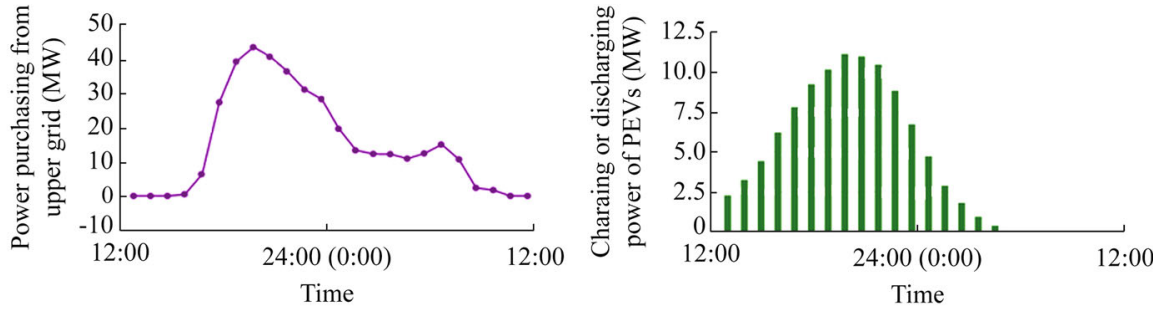

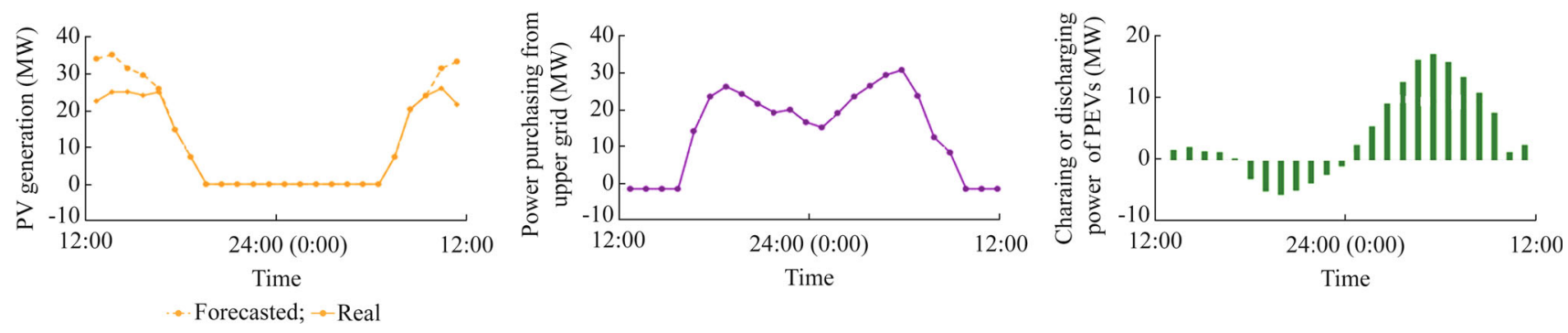

Fig. 7 Energy management results with S3

Table 2 Economic benefit in three scenarios

\begin{tabular}{ll}
\hline Scenario & Total economic benefit (Yuan) \\
\hline S1 & 160156 \\
S2 & 166695 \\
S3 & 183997 \\
\hline
\end{tabular}

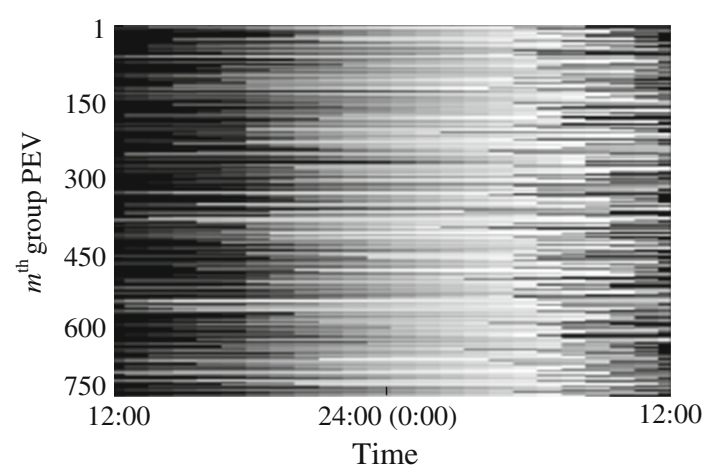

Fig. 8 SOC evolutions of PEVs in S2

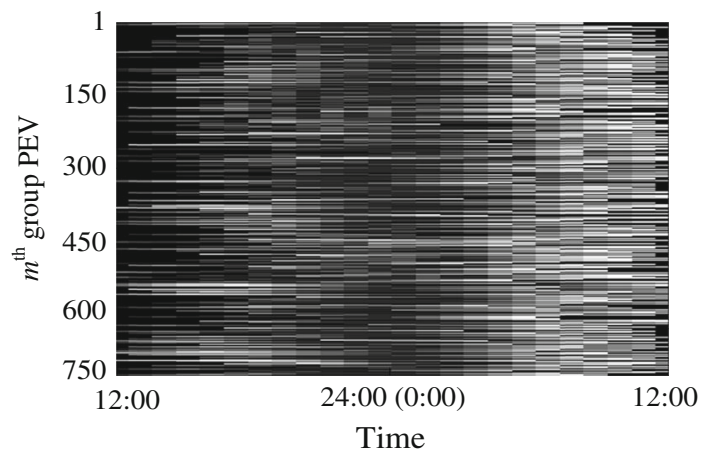

Fig. 9 SOC evolutions of PEVs in S3

to 3:00, and this phenomena can be interpreted that PEVs are arranged to discharge their battery in peak hours and charge to the expectation level later in S3 which can also be seen in Fig. 7, while the battery state would stay at a high level as long as being charged in S2.
The active management model proposed is from the perspective of the local power company, which is mainly focused on achieving the maximization the economic benefit by active generation curtailment of PVs and flexible dispatch of PEVs, and the consumer utility are not included at present. However, PEVs can also get profit through participating in the $\mathrm{V} 2 \mathrm{G}$ program for providing power support during the peak hours, which is considered in S3. At the same time, discharging at peak hours and charging at non-peak hours can improve the load curves, so it is an equivalent win-win situation for both the local power company and the owners of PEVs.

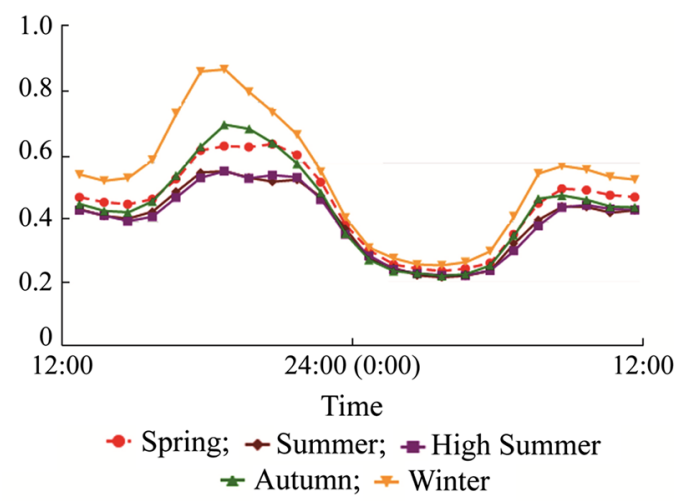

(a) Class 1-DUC weekday templates

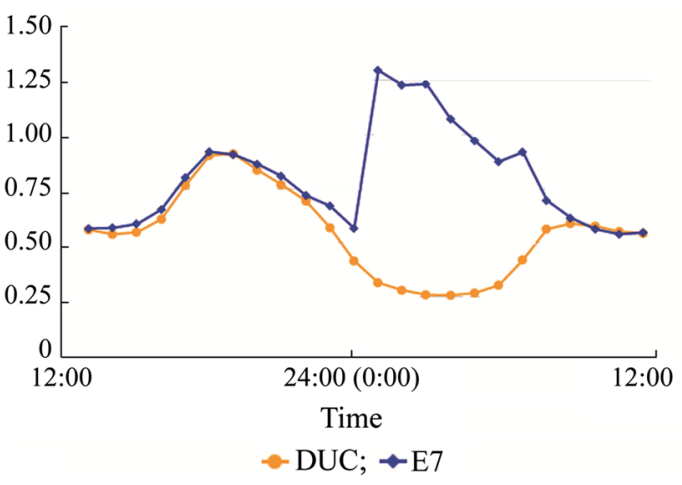

(b) Class 2-E7 weekday templates compared with DUC

Fig. 10 Generic load profile templates 


\subsection{Impact analysis according to different load profiles}

In the previous study, the load is generated according to the basic residential load profile template, and arriving time and departure time of PEVs are simulated based on the basic residential living habits on weekdays. Most people would leave for work around time period 8:00-9:00 and be back home around time period 17:00-18:00. The load mode can be regarded as the Class1-domestic urestricted customer (DUC), according to the classification of the generic UK ELEXON load profiles, whose detailed templates can be found in [20]. The load template used in Sect. 3.2 can be regarded as a sample belonging to that class. Regarding the DUC load template, the trends in different seasons and days (weekday or weekend) are different. So in this section, some other load templates are used and tested based on the proposed active models and strategy to analyze their impacts on the results.

The generic Class1 DUC weekday load templates in different seasons are depicted in Fig. 10a. The load fluctuates severely in winter with a large peak from 16:00 to 21:00, whereas the load profile in summer is much more flat and smooth. Assuming that the initial data of PEVs are the same as that in Sect. 3.2. Based on the summer and winter load profiles, the active model with $\mathrm{S} 3$ is simulated, and schedules are illustrated in Fig. 11a-b.

As it is shown in Fig. 11a-b, the curve shapes of the energy management results are resemble with the winter and summer templates. However in detail, due to the quantities of the loads in winter and summer are different, the power needs are various in different time periods, thus leading to slight difference of PV power generation and PEVs charging/discharging schedules. Specifically, since the load in winter is larger than that in summer, typically in some time periods, PVs can obtain more utilization in winter. There is no PV generation curtailment occurrence in winter from the time period 16:0-17:00, an hour earlier than that in summer. Besides, in the time period 8:00-9:00, no curtailment occurred in winter, one hour later than that in summer. On the other hand, PEVs provide more energy during the time periods from about 16:00 to 24:00 in winter to compensate the peak load compared with summer. Meanwhile, PEVs start charging from time period 0:00-1:00, an hour earlier than that in summer, to guarantee the battery constraints as well.
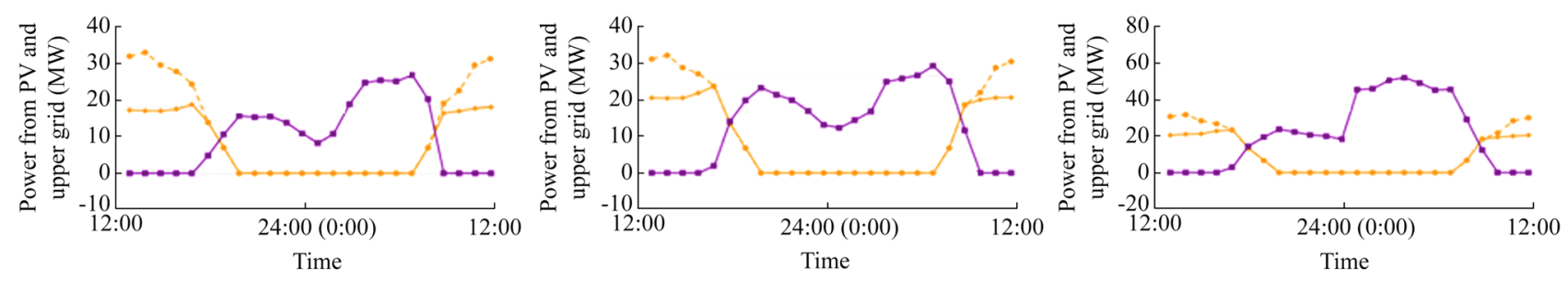

- Forecasted power from PV; - - Real power from PV; - Power from upper grid
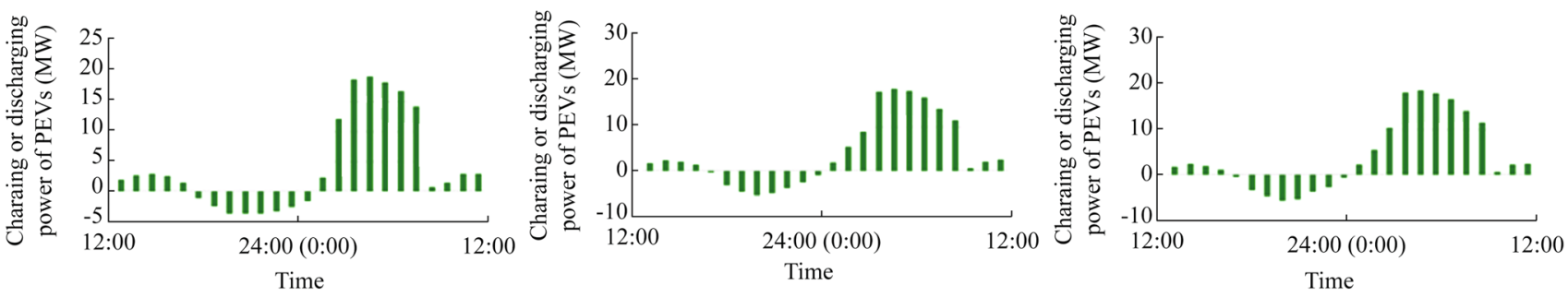

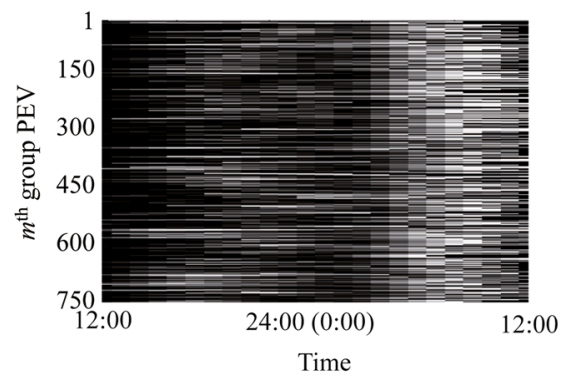

(a) DUC summer weekday

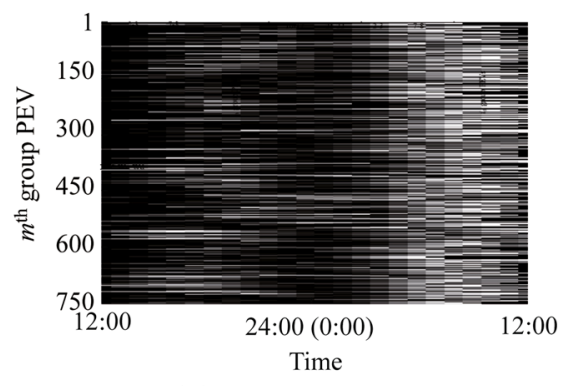

(b) DUC winter weekday

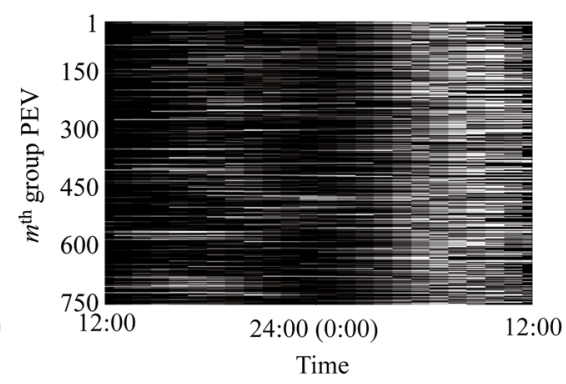

(c) E7 winter weekday

Fig. 11 Energy management results with different load profiles 
Apart from DUC, there exists another domestic type in the generic ELEXON load profiles, called the Economic 7 (E7) as Class 2. And the winter load templates of Generic profile Class 2-E7, compared with that of DUC, are illustrated in Fig. 10b. As shown in the figure, the curves are quite different, especially during the time periods from 23:00 to 8:00, due to widely use of electric heaters during that time periods. And the active management results with E7 type are shown in Fig. 11c.

Compared with Fig. 11a, b, the power purchased from the upper grid in Fig. 11c is almost getting to the limit in the time period 3:00-4:00 in this case for the sake of the larger load demand. The energy management of PV and PEV in Fig. 11c is similar to that of Fig. 11b for the full utilization of PVs and discharging ability of the PEVs considering the available dispatching capacity and the constraints.

\section{Conclusion}

The active energy management strategy can effectively dispatch various low-carbon distributed resources and realize the economic operation of the ADS. A local distribution system is adopted as the test system and an active management model and strategies are presented from the perspective of achieving maximum economic benefits for the local power company. The active features of the ADS, are mainly reflected by the active generation curtailment of renewable DGs considering the operational constraints of the distribution system, as well as PEVs with flexible charging/discharging schedules. The conclusion can be drawn from the case study: the total economic benefits are improved by introducing the intelligent interruptible charging and discharging strategies. Based on different load templates, cases are also simulated and analyzed. Results indicate that the energy management schedules are strongly correlated with the shape and intensity features of the load profile.

It is worth noting that the active management model aiming at maximization of economic benefit is only from the perspective of the distribution company, thus sophisticated behavior models of different aggregators and interactive models between different utilities are not considered yet. Besides, the effects on different parameters, e.g., the initial SOC distribution, and the cost benefit analysis of distributed resources in active management will be studied in the future.

Acknowledgments This work was supported by National High Technology Research and Development Program of China (No. 2014AA051901) and the National Science Foundation of China (No. $51377111)$.
Open Access This article is distributed under the terms of the Creative Commons Attribution 4.0 International License (http:// creativecommons.org/licenses/by/4.0/), which permits unrestricted use, distribution, and reproduction in any medium, provided you give appropriate credit to the original author(s) and the source, provide a link to the Creative Commons license, and indicate if changes were made.

\section{References}

[1] Pilo F, Jupe S, Silvestro F et al (2008) Planning and optimisation of active distribution systems - an overview of CIGRE Working Group C6.19 activities. In: Proceedings of the CIRED 2012 workshop on integration of renewables into the distribution grid, Lisbon. 29-30 May 2012

[2] Chowdhury S, Chowhury SP, Crossley P (2009) Microgrids and active distribution networks. IEE, Stevenage

[3] You Y, Liu D, Yu WP et al (2012) Technology and its trends of active distribution network. Autom Electr Power Syst 36(18):10-16 (in Chinese)

[4] Capitanescu F, Ochoa LF, Margossian H et al (2015) Assessing the potential of network reconfiguration to improve distributed generation hosting capacity in active distribution systems. IEEE Trans Power Syst 30(1):346-356

[5] Alnaser SW, Ochoa LF (2015) Advanced network management systems: a risk-based AC OPF approach. IEEE Trans Power Syst 30(1):409-418

[6] Olivier F, Aristidou P, Ernst D et al (2015) Active management of low-voltage networks for mitigating overvoltages due to photovoltaic units. IEEE Trans Smart Grid. doi:10.1109/TSG. 2015.2410171

[7] Chen SX, Gooi HB, Wang MQ (2012) Sizing of energy storage for microgrids. IEEE Trans Smart Grid 3(1):142-151

[8] Award ASA, El-Fouly THM, Salama MMA (2015) Optimal ESS allocation for load management application. IEEE Trans Power Syst 30(1):327-336

[9] Macedo LH, Franco JF, Rider MJ et al (2015) Optimal operation of distribution networks considering energy storage devices. IEEE Trans Smart Grid. doi:10.1109/TSG.2419134 (to be published)

[10] Green RC, Wang LF, Alam M (2011) The impact of plug-in hybrid electric vehicles on distribution networks: a review and outlook. Renew Sustain Energy Rev 15(1):544-553

[11] Clement-Nyns K, Haesen E, Driesen J (2010) The impact of charging plug-in hybrid electric vehicles on a residential distribution grid. IEEE Trans Power Syst 25(1):371-380

[12] Pieltain Fernández L, Román TGS, Cossent R et al (2011) Assessment of the impact of plug-in electric vehicles on distribution networks. IEEE Trans Power Syst 26(1):206-213

[13] Yang HM, Chung CY, Zhao JH (2013) Application of plug-in electric vehicles to frequency regulation based on distributed signal acquisition via limited communication. IEEE Trans Power Syst 28(2):1017-1026

[14] Zheng JH, Dai MT, Zhang M et al (2012) Load cluster characteristic and modeling of EV charge station in residential district. Proc CSEE 32(22):32-38 (in Chinese)

[15] Taylor J, Maitra A, Alexander M, et al (2009) Evaluation of the impact of plug-in electric vehicle loading on distribution system operations. In: Proceedings of the IEEE PES general meeting (PES'09), Calgary. 26-30 July 2009

[16] Tian LT, Shi SL, Jia Z (2010) A statistical model for charging power demand of electric vehicles. Power Syst Technol 34(11):126-130 (in Chinese) 
[17] Yao WF, Zhao JH, Wen FS et al (2012) A charging and discharging strategy for electric vehicles based on bi-level optimization. Autom Electr Power Syst 36(11):30-37 (in Chinese)

[18] Tomic J, Kempton W (2007) Using fleets of electric-drive vehicles for grid support. J Power Sour 168(2):459-468

[19] Andersson SL, Elofsson AK, Galus MD et al (2010) Plug-in hybrid electric vehicles as regulating power providers: case studies of Sweden and Germany. Energy Policy 38(6):2751-2762

[20] Load profile classes. ELEXON.co.uk, UK

[21] United Kingdom Generic Distribution System (UKGDS). The Centre for Sustainable Electricity and Distributed Generation, UK

[22] Liu TQ, Jiang DL (2012) Economic operation of microgrid based on operation mode optimization of energy storage unit. Power Syst Technol 36(1):45-50 (in Chinese)

[23] GAMS: a user's guide. General Algebraic Modeling System (GAMS) Development Corporation, Washington, DC

Yue XIANG received the B.S. degree from the School of Electrical Engineering and Information, Sichuan University, China, in 2010, where he is currently pursuing the Ph.D degree. His main research interests are power system planning and optimal operation, renewable energy integration, and smart grids.

Junyong LIU received the Ph.D degree from Brunel University, UK, in 1998. He is a professor in the School of Electrical Engineering and Information, Sichuan University, China. His main research areas of interest are power system planning, operation, stability and computer applications.

Wei YANG received the B.S. degree from the School of Electrical Engineering and Information, Sichuan University, China, in 2012, where he is currently pursuing the Ph.D degree. His main research interests are electric vehicle integration and smart grids.

Cheng HUANG received the B.S. and M. S. degree from the College of Electronics and Information Engineering, Sichuan University, China, in 2010 and 2013, respectively, where he is currently pursuing the Ph.D degree. His main research interests are information system security and computer applications. 